each year, for children aged $<15$ years, admitted to hospital with severe complications of laboratory proven influenza.

Results A total of 489 cases were reported: median age $=3$ years (0-14.8) and 56.5\% were boys. Most 338 (69\%) had influenza A. Complications included pneumonia (61.2\%), encephalitis (13.3\%), myocarditis/pericarditis (3.7\%), shock (3.9\%), rhabdomyolysis (3.9\%). Viral or bacterial co-infections were reported in $18 \%$. Of the 489 children 24 (5.3\%) were vaccinated for influenza and of 174 of children who had chronic conditions pre-disposing for influenza 15 (8.2\%) were vaccinated. There were 21 deaths. Deaths occurred in each year of surveillance except for 2016, and in 52.3\% the children had a pre-existing condition (e.g.rare genetic syndromes, cerebral palsy, ulcerative colitis), but $47.6 \%$ of children that died were previously healthy. Given that almost half of the children who died were previously healthy, there is a need for rapid diagnosis and treatment of children with severe complications of influenza, and all children should be vaccinated for influenza, not just those who have underlying chronic conditions.

Conclusions Awareness raising and education about early diagnosis and treatment in addition to increasing annual influenza vaccination is neded among health professionals caring for children children whether or not the children have pre-disposing medical conditions. Ongoing surveillance is needed to monitor the effectivenes of vaccination programmes to prvent these seious outcomes of influenza infection in children.

\section{P7 STANDARDISED PROCEDURE TROLLIES SAVE TIME AND STRESS - A QI PROJECT}

D Maxted, A Dewar, M Moran. Department of Paediatrics, Nottingham Children's Hospital, Nottingham University Hospitals NHS Trust, Nottingham, UK

\subsection{6/archdischild-2018-rcpch.7}

Introduction Basic procedures such as phlebotomy and cannulation are commonly performed in children admitted to hospital by junior doctors. In our tertiary teaching hospital, children are admitted to one of seven wards after initially been seen on the admission unit. Out of hours junior doctors cover a number of wards, often attending wards not worked on in-hours. We highlighted a problem that finding equipment was frequently proving difficult and an in-effective use of time; partly due to varying equipment locations on each ward. We set out to streamline the process by using a standardised procedure trolley.

Methods We surveyed junior doctors to identify equipment that would be used frequently enough to form our prototype trolley. We then timed junior doctors finding a list of equipment using the prototype trolley, and without the trolley on a ward they were familiar working on and on a ward they haven't been before. Following this we agreed with ward managers to roll out the standard trolley to five wards.

Results Junior doctors on unfamiliar wards found the required equipment after an average of $10 \mathrm{~min} 13 \mathrm{~s}$ (6 min 28-15 m 26). On a familiar ward this reduced to $5 \mathrm{~min} 31 \mathrm{~s}(2 \mathrm{~m} \mathrm{49-}$ $9 \mathrm{~m} \mathrm{18}$ ). Using our prototype trolley the average reduced further to $1 \mathrm{~min} 35 \mathrm{~s} \mathrm{(} 1 \mathrm{~m} 24-1 \mathrm{~m} \mathrm{46}$ ). On our unit, 30 procedures were carried out in 24 hours; over 200 in a week. Reducing the average time from $8 \mathrm{~min} 17 \mathrm{~s}$ (combing unfamiliar and familiar results) to $1 \mathrm{~min} 35 \mathrm{~s}$ would save $6 \mathrm{~min} 42 \mathrm{~s}$ per procedure. This would result in over 20 hours of junior doctor time saved in a single week. Post roll out audit found 3 to be well stocked, 1 to be adequately stocked and 1 poorly stocked. This correlates with our survey of doctors; just over a third felt trollies didn't have enough equipment stocked on it. 94\% felt the trollies saves time when stocked!

Conclusions We highlighted a problem with undertaking a common procedure in our patients. We designed a solution, tested its efficacy and engaged stakeholders in its implementation. We have shown how a simple solution could save over 20 hours of junior doctor time a week, improving not only patient care but junior doctor satisfaction.

\section{P8 1 BONE MINERAL DENSITY IN CHILDREN AND ADOLESCENTS WITH CYSTIC FIBROSIS, SHOULD WE BE DOING LESS MONITORING?}

${ }^{1}$ CF O'Toole, ${ }^{2,3} \mathrm{~A}$ Bush, ${ }^{2,3} \mathrm{SB}$ Carr. ' Sixth Form, Royal Grammar School, Guildford, UK; ${ }^{2}$ Department of Paediatrics, Imperial College, London, UK; ${ }^{3}$ Department of Paediatrics, Royal Brompton Hospital, London, UK

\subsection{6/archdischild-2018-rcpch.8}

Background Cystic fibrosis (CF) is a multi-system disease resulting from mutations in the CFTR gene. CF patients are at risk of developing osteopenia. Regular monitoring of bone mineral density (BMD) is currently recommended in the standards of care for CF.

Aims

- To explore the extent of low BMD in children with CF and identify risk factors associated with decreased BMD.

- Establish the rate of decline in BMD to inform future practice.

Methods All children undergo routine DEXA scan at bi-annual assessments from 8 years of age. A single centre retrospective review of CF Registry records and online hospital records was conducted for children born 2000-2006. Z-score values were obtained for BMD and BMI as well as $\mathrm{FEV}_{1} \% / \mathrm{FVC} \%$ predicted (GLI), and possible confounding factors were also recorded.

\begin{tabular}{llllll}
\multicolumn{6}{l}{ Abstract P8 Table 1} \\
\hline DEXA sequence & Scan 1 & Scan 2 & Scan 3 & Scan 4 & ANOVA sig. \\
\hline Number & 96 & 87 & 60 & 18 & \\
Age in years (Mean,SD) & $9.98(1.9)$ & $11.79(1.8)$ & $13.24(1.5)$ & $14.34(1.5)$ & \\
M/F & $37 / 59$ & $33 / 54$ & $25 / 35$ & $8 / 10$ & \\
L1-L4 & -0.12 & -0.37 & -0.42 & -0.96 & 0.008 \\
L2 & -0.27 & -0.48 & -0.59 & -1.13 & 0.013 \\
FEV1\% & $86 \%$ & $83 \%$ & $80 \%$ & $78 \%$ & 0.174 \\
BMI Z-Score & -0.03 & -0.05 & 0.04 & 0.18 & 0.844 \\
Vit-D & 72 & 69 & 71 & 78 & 0.577 \\
\hline
\end{tabular}

Results 96 children (59 female) had a first DEXA scan at a mean age of 10 ( \pm 1.94 years). 262 DEXA scans were performed with a median of 2 per individual. Thirteen (13.5\%) had an abnormal DEXA scan over the course of the study; only 2 of these were age $<10$ years $(n=48$ performed in this age range). Risk factors for abnormal BMD (z-score $\leq-2$ ) were: a BMI $z$-score $\leq 2 ;>50$ days of intravenous antibiotics in the year before the scan; and $\mathrm{FEV}_{1} \%<50$. The table shows 
mean results for sequential scans; rate of decline was unpredictable. The mean change per year was -0.1 z-scores $(S D=$ \pm 0.5 ). Neither Vit D levels nor the CF-related diabetes was related to baseline or changes in BMD.

Conclusion Low lung function and BMI $z$-scores were the greatest indicators of being at risk of osteopaenia for the cohort. With a rate of decline of only $0.1 \mathrm{z}$-score per annum the frequency of DEXA scans could be decreased in low risk children.

\section{P9 A RANDOMISED, DOUBLE-BLIND, PARALLEL GROUP, PLACEBO-CONTROLLED TRIAL OF METFORMIN IN TUBEROUS SCLEROSIS COMPLEX}

'S Amin, ${ }^{1} \mathrm{AA}$ Mallick, ${ }^{1} \mathrm{H}$ Edwards, ${ }^{1} \mathrm{~A}$ Lux, ${ }^{2} \mathrm{M}$ Laugharne, ${ }^{1} \mathrm{M}$ Likeman, ${ }^{3} \mathrm{~A}$ Khan, ${ }^{4} \mathrm{~F}$ O'Callaghan. ${ }^{\text {P}}$ Paediatric Neurology, University Hospitals Bristol, Bristol, UK; ${ }^{2}$ Radiology, Royal United Hospitals Bath, Bath, UK; ${ }^{3}$ Dermatology, University Hospital North Durham, Durham, UK; ${ }^{4}$ Neurosciences, University College London, London, UK

\subsection{6/archdischild-2018-rcpch.9}

Introduction Tuberous Sclerosis Complex (TSC) is a genetic disorder characterised by the development of benign tumours, secondary to the loss of inhibitory regulation of the mTOR (mammalian Target Of Rapamycin) intracellular growth pathway. Metformin inhibits the mTOR pathway. We investigated the effect of Metformin in patients with TSC.

Methods This was a multicentre randomised, double-blind, parallel group, placebo-controlled trial of metformin in children and adults with TSC. Patients were randomly allocated to placebo or metformin (1:1) for 12 months. The main outcomes were percentage volume change of renal angiomyolipomas (AML) and cerebral Subependymal Giant Cell Astrocytomas (SEGA). Blinded MRI assessments of AMLs and SEGAs were performed at baseline and at 12 months.

Results Fifty-one patients participated, of whom 43 were adults (age range 10-50 years, median age 27, IQR 17). 24 received placebo and 27 received metformin.

The mean AML volume increase from baseline was 25.5\% for the placebo group and $9.6 \%$ for the metformin group. Difference in response, $15.9 \%$ (95\% CI $-9 \%$ to $41 \%$ ) $\mathrm{p}=0.221$.

Twenty-seven patients had SEGAs: 13 received placebo and 14 metformin. The mean SEGA volume increased from baseline by $37 \%$ in the placebo group but reduced from baseline by $23.3 \%$ in the metformin group. Difference in response, $60.3 \%$ (95\% CI $-0.4 \%$ to $111, \mathrm{p}=0.048)$.

Three serious adverse events occurred that reflected the underlying disease. Two were AML haemorrhages and one was worsening seizures requiring hospitalisation.

Conclusions Metformin is safe and well tolerated in children and adults with TSC. Patients on metformin had a significant reduction in SEGA volume compared with placebo. Metformin did not reduce AML size but growth appeared slower than in the placebo group, although this difference was not statistically significant. There may be a role for metformin in slowing or reversing the growth of life-threatening hamartomas in TSC. Further study is justified.

\section{Young People's Health Special Interest Group}

\section{G10 ARE WE USING THE HEEADSSS ASSESSMENT TO SCREEN FOR PSYCHOSOCIAL RISKS WHEN YOUNG PEOPLE ARE ADMITTED TO HOSITAL?}

AE Richardson, M Cooper, DM Wood. Department of Paediatrics, Nottingham Children's Hospital, Nottingham University Hospitals, Nottingham, UK

\subsection{6/archdischild-2018-rcpch.10}

Aims To evaluate the use of an adolescent psychosocial screening tool (HEEADSSS assessment) on admission to hospital. To identify barriers to screening and inform service development. Methods A retrospective evaluation of a systematic sample of 10-18 year olds admitted over a six month period. Case notes were reviewed to identify the recording of the components of the HEEADSSS assessment. We included an equivalent number of young people admitted with mental health as well as physical health presentations. We asked the medical team to identify barriers to using the HEEADSSS tool when admitting young people to the acute paediatric ward via an electronic survey.

Results We identified 60 young people (mean 13.9 years), 30 admitted with a mental health presentation, and 30 with 'other' acute presentations. Overall 25\% (15/60) had a fully completed HEEADSSS tool, with 57\% (34/60) partially completed, and absent in $18 \%(11 / 60)$. In young people presenting with mental health problems $47 \%$ (14/30) had a completed HEEADSSS, occurring in only $3 \%$ ( $1 / 30$ patient) in the 'other' category (chi squared $15.02 \mathrm{p}<0.0001$ ). In the 'other' group $18 / 30(60 \%)$ had a partially completed HEEADSSS, and absent in 37\%. Of those presenting with a 'mental health' problem, $16 / 30$ (53\%) had a partially completed assessment (Fisher's Exact Test $p=0.0001$ ). Overall the components least frequently documented were Eating (13\%), Safety (30\%), Activities (35\%) and Sex (37\%). Barriers to completion included family members being present, the engagement of the young person, lack of familiarity/training using HEEADSSS, and the hospital environment.

Conclusion Our results showed adolescent psychosocial screening is only completed in 1 in 4 young people. The proportion of completed screening was higher in young people presenting with mental health problems compared to those with other presentations. These differences remain when partially completed screens are included. Some parts of the assessment were more frequently completed than others. Barriers to use by admitting clinicians included factors relating both to clinician knowledge and skills, and the hospital environment. These results will assist in clinician training as well as quality improvement for young people admitted to hospital. 